\title{
Clinical correlation and antimicrobial susceptibility pattern of Chryseobacterium spp.: A three year prospective study
}

\author{
Vishwanath Singh Yadav, Bimal Ku Das*, Sarita Mohapatra, M Nizam Ahmed, \\ Hitender Gautam, Arti Kapil, Seema Sood, Benu Dhawan, Rama Chaudhry \\ Department of Microbiology, All India Institute of Medical Sciences, New Delhi, India.
}

SUMMARY Chryseobacterium species are widely distributed in the environment. They are rarely found in hospital settings causing nosocomial infections. Limited data is available regarding their epidemiology, clinical significance and antimicrobial susceptibility patterns. This study was aimed to identify different species of Chryseobacterium using matrix-assisted laser desorption/ionization time of flight mass spectrometry (MALDI-TOF MS) and to correlate clinically with antimicrobial susceptibility patterns in a tertiary care hospital in north India. We also performed phenotypic tests, which may be useful to differentiate this bacterium from other non-fermenters. A total of 20 isolates of Chryseobacterium spp. were identified over a period of 3 years. Chryseobacterium indologenes $(18 / 20)$ was the most common species isolated followed by Chryseobacterium gleum (2/20) from various clinical samples. Antimicrobial susceptibility testing (AST) was performed. Susceptibility to rifampicin was observed at a maximum (75\%) followed by piperacillin-tazobactum $(45 \%)$. Susceptibility against imipenem, meropenem, cotrimoxazole and cefoperazone-sulbactum were observed approximately $33 \%$. Amikacin, cefotaxime and ceftazidime showed least susceptibility results. Further clinical correlation was established.

Keywords Chryseobacterium indologenes, Chryseobacterium gleum, nosocomial infections, MALDI-TOF MS

\section{Introduction}

Chryseobacterium species are among the emerging pathogens causing infections in humans. They are rarely isolated from clinical samples (1). There are more than 100 species of Chryseobacterium identified until now (2). However, only Chryseobacterium indologenes, Chrysebacterium gleum and Chryseobacterium hominis are proven to cause infections in humans. They are commonly found in soil and water environments. They often colonize in water suppliers, taps, and sink basins; thereby forming reservoirs for infections in hospital environments.

Colonization of various medical devices containing fluids such as respirators, humidifiers, incubators for newborns, syringes, etc. have been well documented (3-5). Moreover, devices such as prosthetic valves and intravascular catheters have also been reported as a source of infection for this bacterium (6). In few other clinical settings, Chryseobacterum spp. have been described as causative agents of meningitis, pneumonia, endocarditis, bacteremia, infections of skin and soft tissue, and ocular infections (7-13). Primarily, it is an opportunistic pathogen that infects newborns and immunocompromised individuals of different age groups (14).

There are limited data published on the clinical significance and antimicrobial susceptibility of Chryseobacterium spp. worldwide including India (15). Breakpoints are not available for this newly emerging pathogen. Our study aims to find the clinical significance and antimicrobial susceptibility pattern of different Chryseobacterium spp. isolated from various clinical specimens.

\section{Materials and Methods}

This was a prospective study conducted in the Department of Microbiology of a tertiary care hospital in North India. The duration of the study was 3 years from January 2017 to December 2019. Ethical clearance was obtained from the institute ethics committee. In this study, we aimed to find the recent trends and clinical correlation of various Chryseobacterium species in different clinical specimens.

\subsection{Specimen collection}

Isolates from consecutive clinical samples (blood, CSF, 


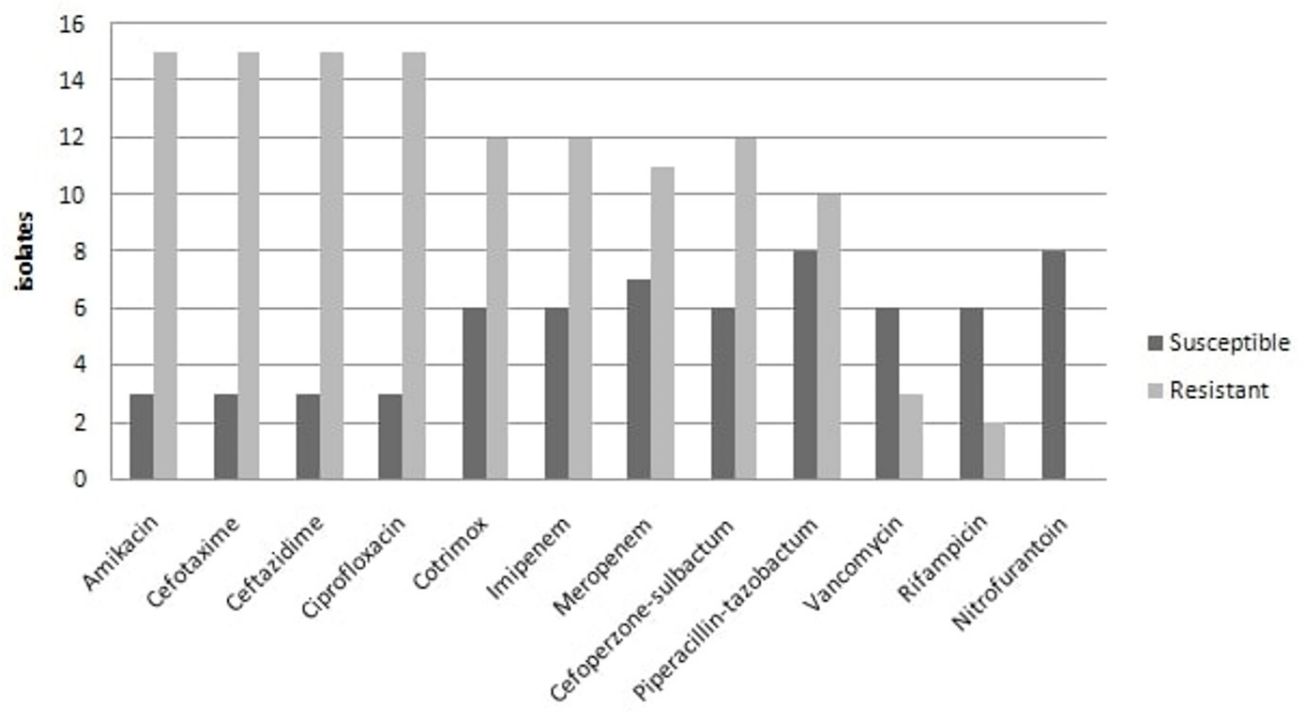

Figure 1. Antimicrobial susceptibility patterns of $C$. Indologenes isolates.



Figure 2. Dark-yellow colored colonies of $C$. indologeneson blood agar and CLED media.

urine, pus, respiratory samples, stool, pleural, peritoneal, pericardial fluids, bone marrow aspirates, catheter tips, etc.) had been processed for aerobic culture in the bacteriology laboratory during the defined period using standard operating procedures. Then final identification of the growth from culture was carried out using MALDI-TOF MS. Phenotypic characterization was also done using standard biochemical tests.

\subsection{Drug susceptibility pattern}

Antimicrobial susceptibility testing of 18 isolates of C. indologenes was performed on Muller Hinton agar (MHA) by Kirby Bauer disc diffusion method (Figure 1). SENTRY antimicrobial surveillance program was only the largest report published that showed data of 5 years (1997-2001) including antimicrobial susceptibility testing of Chryseobacterium spp. Because no standard guidelines for reporting Antimicrobial Susceptibility Testing (AST) for Chryseobacterium spp. were available, we performed AST using antimicrobial agents recommended by the SENRY antimicrobial surveillance program and the result was interpreted using break points for Pseudomonas aeruginosa, Staphylococcus aureus (for interpretation of cotrimoxazole, rifampicin, nitrofurantoin) and Enterococcus spp. (for interpretation of vancomycin) as recommended by the Clinical and Laboratory Standards Institute (CLSI) 2017 guidelines (17).

\subsection{Clinical correlation}

All the cases were prospectively followed to establish clinical correlation. Any changes in treatment/discharge/ death were noted in the data sheet.

\section{Results}

A total of 20 isolates (Chryseobacterium indologenes, $n=18$; and Chryseobacterium gleum, $n=2$ ) were identified from various clinical specimens during the study period. Among the 18 isolates of $C$. indologenes, nine were isolated from blood, eight were from urine and one was from a BAL (bronchoalveolar lavage) specimen. Two C. gleum species were isolated from blood and BAL samples, respectively. Phenotypic identification of all isolates was also performed using conventional methods. The colonies on blood agar were observed to be yellowish in color, 1-2 mm, non-hemolytic, circular, lowconvex and translucent with entire margins. No growth was observed on MacConkey agar. Urine samples were processed on CLED (Cysteine Lactose Electrolyte Deficient agar) media, which showed similar yellow colonies as on blood agar (Figure 2). The organism was found to be gram-negative non-motile bacilli with catalase positive and oxidase positive reactions. The Hugh and Leifson oxidative fermentative (OF) test showed an oxidative reaction. Indole was produced in tryptophan broth; whereas methyl red, urease production and citrate utilization tests were negative. C. gleum can be distinguished from $C$. indologenes by their ability to reduce nitrite to nitrogen. Chryseobacterium spp. can be easily differentiated phenotypically from other non- 
Table 1. Clinical details of patients showing culture of $C$. indologenes

\begin{tabular}{lcccc}
\hline Specimen & $\begin{array}{c}\text { No. of Chryseobacterium spp. } \\
(n=18)\end{array}$ & $\begin{array}{c}\text { Medical Device } \\
(n=10)\end{array}$ & Comorbidities & Positive in Repeat isolation \\
\hline Urine & $8(44 \%)$ & $3(37.5 \%)$ & $4(50 \%)$ & $3(37.5 \%)$ \\
Blood & $9(50 \%)$ & $6(66.6 \%)$ & $3(33 \%)$ & $1(11 \%)$ \\
BAL & $1(5.5 \%)$ & $1(100 \%)$ & 0 & Nil \\
\hline
\end{tabular}

Medical device: Central line venous cather, Ventillator, urinary catheter. Comorbidities: Malignancy, immunosuppression.

fermenters as they are non-motile GNBs producing yellow colored colonies on blood agar, indole positive and inability to grow on MacConkey agar.

The patients, whose clinical specimen showed growth of Chryseobacterium spp. were prospectively followed up for their underlying conditions, co-morbidities, presence of indwelling devices, antibiotic history, change in therapeutic management (based on the culture and AST report), outcome, etc. More than $70 \%$ of the patients had at least one or more mechanical devices (i.e. urinary catheter, central venous catheter, or endotracheal tube) at the time of identification of infection by Chryseobacterium spp. (Table 1).

The susceptibility to rifampicin was observed as the highest (75\%) followed by piperacillin-tazobactum (45\%). Susceptibility against imipenem, meropenem, cotrimoxazole and cefoperazone-sulbactum were observed to be approximately $33 \%$. Amikacin, cefotaxime and ceftazidime showed a least susceptibility pattern. All the 8 isolates of $C$. indologenes isolated from urine specimens were susceptible to nitrofurantoin. The AST of C. gleum showed maximum susceptibility to piperacillin-tazobactum and cefoperazone-sulbactum followed by cotrimoxazole, cefotaxime, imipenem and meropenem.

The isolation of $C$. indologenes was clinically significant among 4 out of $18(22.2 \%)$ patients (three from urine samples and one from blood). The isolates from the urine samples were identified from acute myeloid leukemia (AML) patients and there was presence of the same growth on repeated occasions. Among the three patients, two of them had only fever and one of them had a urinary catheter in situ with urinary symptoms. Isolates from these three patients were susceptible to nitrofurantoin only. All of them were started on oral nitrofurantoin treatment after the susceptibility reports were generated. There was satisfactory clinical improvement with nitrofurantoin in all three patients and subsequent samples from these patients were sterile after completion of antibiotic treatment. A blood sample from central venous catheter in an elderly patient with acute kidney injury showed growth of $C$. indologenes on repeated occasions. On the basis of AST report, piperacillin-tazobactum was started and patient showed clinical improvement but unfortunately, he expired 4 days later due to multi-organ failure.

The two C. gleum strains were isolated from blood and BAL samples. Repeated blood cultures were found sterile. The isolate from blood was sensitive to all relevant antimicrobials except amikacin. An isolate from a BAL sample was sensitive to Cefoperazone-sulbactum and Piperacillin-tazobactum. The clinical significance of these isolates could not be established.

\section{Discussion}

Chryseobacterium spp. are emerging gram-negative bacilli belonging to the family of non-fermenters. With the use of MALDI-TOF MS, Chryseobacterium spp. are being increasingly identified. Since it exists in the hospital environment, a positive growth in culture has doubtful clinical significance. Chryseobacterium infections have been seen in patients with comorbidities like diabetes mellitus, chronic kidney disease, cardiovascular disease, chronic obstructive pulmonary disease and malignancies (16). Beside colonization in various medical devices by formation of bio-films other virulence factors like protease activity are yet to be explored and require further studies to establish their role in pathogenesis $(14,16)$. There are limited data available determining the pathogenic role of Chryseobacterium spp. The SENTRY study (1997 to 2001) estimated the epidemiology and antimicrobial susceptibility pattern of Chryseobacterium infections worldwide, where the most common isolated species was $C$. meningosepticum followed by C. indologenes and C. gleum (15). The investigators described the differences in clinical features and anti-microbial susceptibility patterns between $C$. indologenes and C. gleum (16). They analyzed the database from 2005 and 2017 to identify patients with Chryseobacterium infections. A total of 84 isolates of C. indologenes and 42 isolates C. gleum were identified and studied during this period. Our study results were in agreement with this. There are no specific antimicrobial susceptibility testing guidelines available either from CLSI or EUCAST for the genus Chryseobacterium.

In our study, the most common species isolated was C. indologenes followed by C. gleum. C. indologenes was clinically correlated in $22 \%$ of the total patients who improved after changing the antibiotic regime. Urinary catheter remains an important risk factor for the causation of UTI by $C$. indologenes in most of the previously published literature. In the current study, two symptomatic patients without a urinary catheter showed significant growth of $C$. indologenes 
on repeated occasions. Moreover, both the patients were immunosuppressed, which signifies there might be an increased chance of acquisition of infection in immunocompromised states. Because the numbers are very low in our study, follow up studies with adequate numbers may be helpful. In the current study, all urinary isolates observed were sensitive to nitrofurantoin, which is in agreement with a study conducted by Taneja et al. (17). More studies are needed to establish the efficacy of nitrofurantoin against this species.

A study by Kirby et al. showed more than $90 \%$ susceptibility of $C$. indologenes to piperacillintazobactum and cotrimoxazole (15). In our study, the susceptibility was $45 \%$ and $33 \%$ for piperacillintazobactum and cotrimoxazole, respectively. A recently published study by Lin et al. showed maximum susceptibility to minocycline $(67.9 \%)$ followed by cotrimoxazole $(47.9 \%)$.

Chryseobcterium gleum is also an uncommon pathogen rarely isolated from various clinical samples. There are limited data regarding C. gleum. Only a few case reports were reported from india. A study published by Rajendran et al. showed a case of nosocomial urinary tract infection by $C$. gleum in a diabetic elderly male patient with chronic renal disease and was successfully treated with ciprofloxacin (18). Few case reports published on $C$. gleum in patients suffering from chronic granulomatous diseases were possibly due to infection of the stent $(19,20)$. In the current study, clinical correlation could not be established in both the C. gleum isolates and were considered to be contaminants. Regarding antimicrobial susceptibility patterns of C. gleum, our study showed maximum susceptibility to piperacillintazobactum, which coincides with the study done by Lin and his colleagues, published in 2019 (16).

In conclusion, there is increasing incidence of nosocomial infection due to multidrug resistant Chryseobacterium spp.. Early and accurate diagnosis of Chryseobacterium infection can provide an edge in appropriate management of the critically ill patients. With the help of advanced techniques like MALDI-TOF MS, these new emerging pathogens can be easily and accurately identified. There is a need to determine the breakpoints for various antibiotics against this bacterium across the world, to make a universal consensus for the antimicrobial susceptibility testing of Chryseobacterium species.

\section{Funding: None.}

Conflict of Interest: The authors have no conflicts of interest to disclose.

\section{References}

1. Bhuyar G, Jain S, Shah H, Mehta VK. Urinary tract infection by Chryseobacterium indologenes. Indian J Med Microbiol. 2012; 30:370-372.
2. Parte AC. LPSN - List of Prokaryotic names with Standing in Nomenclature (bacterio.net), 20 years on. Int J Syst Evol Microbiol. 2018; 68:1825-1829.

3. du Moulin GC. Airway colonization by Flavobacterium in an intensive care unit. J Clin Microbiol. 1979; 10:155-160.

4. Hoque SN, Graham J, Kaufmann ME, Tabaqchali S. Chryseobacterium (Flavobacterium) meningosepticum outbreak associated with colonization of water taps in a neonatal intensive care unit. J Hosp Infect. 2001; 47:188192.

5. Lin YT, Jeng YY, Lin ML, Yu KW, Wang FD, Liu CY. Clinical and microbiological characteristics of Chryseobacterium indologenes bacteremia. J MicrobiolImmunol Infect. 2010; 43:498-505.

6. Chou DW, Wu SL, Lee CT, Tai FT, Yu WL. Clinical characteristics, antimicrobial susceptibilities, and outcomes of patients with Chryseobacterium indologenes bacteremia in an intensive care unit. Jpn J Infect Dis. 2011; 64:520-524.

7. Nulens E, Bussels B, Bols A, Gordts B, Van Landuyt HW. Recurrent bacteremia by Chryseobacterium indologenes in an oncology patient with a totally implanted intravascular device. Clin Microbiol Infect. 2001; 7:391-393.

8. Hendaus MA, Zahraldin K. Chryseobacterium indologenes meningitis in a healthy newborn: a case report. Oman Med J. 2013; 28:133-134.

9. Atıc1 S, Ünkar ZA, Erdem K, Kadayifci EK, Karaaslan A, Memişoğlu AÇ, Soysal A, Toprak NÜ, Söyletir G, Özek E, Bakır M. Ventilator-associated pneumonia caused by Chryseobacterium indologenes: a rare infant case and review of the literature. Springerplus. 2016; 5:1741.

10. Bomb K, Arora A, Trehan N. Endocarditis due to Chryseobacterium meningosepticum. Indian J Med Microbiol. 2007; 25:161-162.

11. Bhagawati G, Bhardwaj A, Sajikumar R, Singh SP, Prajapati S. Bacteremia by Chryseobacterium indologenes in a patient with lung cancer: a clinical and microbiological investigation. Indian J Crit Care Med. 2019; 23:157-159.

12. Srinivasan G, Muthusamy S, Raveendran V, Joseph NM, Easow JM. Unforeseeable presentation of Chryseobacterium indologenes infection in a paediatric patient. BMC Res Notes. 2016; 9:212.

13. Bloom AH, Perry HD, Donnenfeld ED, Davis RG. Chryseobacterium meningosepticum keratitis. Am J Ophthalmol. 2003; 136:356-357.

14. Bloch KC, Nadarajah R, Jacobs R. Chryseobacteriu mmeningosepticum: an emerging pathogen among immunocompromised adults. Report of 6 cases and literature review. Medicine (Baltimore). 1997; 76:30-41.

15. Kirby JT, Sader HS, Walsh TR, Jones RN. Antimicrobial susceptibility and epidemiology of a worldwide collection of Chryseobacterium spp.: report from the SENTRY Antimicrobial Surveillance Program (1997-2001). J Clin Microbiol. 2004; 42:445-448.

16. Lin JN, Lai CH, Yang CH, Huang YH. Differences in clinical manifestations, antimicrobial susceptibility patterns, and mutations of fluoroquinolone target genes between Chryseobacterium gleum and Chryseobacterium indologenes. Antimicrob Agents Chemother. 2019; 63:e02256-18.

17. Kaur H, Mohan B, Hallur V, Raj A, Basude M, Mavuduru RS, Taneja N. Increased recognition of Chryseobacterium species as an emerging cause of nosocomial urinary tract infection following introduction of matrix-assisted 
laser desorption/ionisation-time of flight for bacterial identification. Indian J Med Microbiol. 2017; 35:610-616.

18. Rajendran P, Muthusamy S, Balaji VK, Rakesh GJ, Easow JM. Urinary tract infection due to Chryseobacterium gleum, an uncommon pathogen. Indian J PatholMicrobiol. 2016; 59:551-553.

19. Rawat A, Vignesh P, Sharma A, Shandilya JK, Sharma M, Suri D, Gupta A, Gautam V, Ray P, Rudramurthy SM, Chakrabarti A, Imai K, Nonoyama S, Ohara O, Lau YL, Singh S. Infection profile in chronic granulomatous disease: a 23-year experience from a tertiary care center in North India. J Clin Immunol. 2017; 37:319-328.

20. Garg S, Appannanavar SB, Mohan B, Taneja N.
Pyonephrosis due to Chryseobacterium gleum: a first case report. Indian J Med Microbiol. 2015; 33:311-313.

Received July 28, 2020; Revised October 26, 2020; Accepted December 11, 2020.

*Address correspondence to:

Bimal Ku Das, Department of Microbiology, All India Institute of Medical Sciences, New Delhi-110029, India.

E-mail: tezpur.bimal@gmail.com

Released online in J-STAGE as advance publication January 12, 2021. 